\title{
Efecto de fisuras en la tracción de juntas soldadas de acero A36 mediante simulación numérica
}

\author{
Effect of cracks on the tensile strength of A36 steel welded joints by means \\ of numerical simulation
}

Carlos Oswaldo Serrano Aguiar. ${ }^{1}$, Marcelo Esteban Calispa Aguilar. ${ }^{2}$, Marco Antonio Ordoñez Viñan. ${ }^{3} \&$ Luis Santiago Choto Chariguamán. ${ }^{4}$

Recibido: 11-10-2021 / Revisado: 21 -10-2021 /Aceptado: 17-11-2021/ Publicado: 08-12-2021

\begin{abstract}
DOI: https://doi.org/10.33262/concienciadigital.v4i4.2.1948

Introduction: The most critical discontinuities that occur in welding are cracks, showing a high concentration of stresses and therefore a reduction in the loads that can be applied to welded joints, the construction standards for pipeline transportation systems such as API 1104 are very strict regarding the acceptance of these discontinuities, but only with an empirical foundation. Objective: Determine the influence of the length of the cracks on the yield stress of joints welded with ASTM A36 steel, using numerical simulation by finite elements. Methodology: A qualitative analysis was carried out to determine the load that can be applied to pipes joined by welding, the independent variables are the thickness of the pipe, the length of the crack and the diameter of the pipe. Commercial pipes are selected from 101.4 to $508 \mathrm{~mm}$ (4 to 20 inches) in nominal diameter, with 3

\footnotetext{
${ }^{1}$ Escuela Superior Politécnica de Chimborazo, Facultad de Mecánica Riobamba, Ecuador. carlos.serrano@espoch.edu.ec, ORCID: 0000-0002-0653-1115

${ }^{2}$ Escuela Superior Politécnica de Chimborazo, Facultad de Mecánica Riobamba, Ecuador. marcelo.calispa@espoch.edu.ec, ORCID: 0000-0002-4085-8488

${ }^{3}$ Escuela Superior Politécnica de Chimborazo, Facultad de Mecánica Riobamba, Ecuador. marco.ordonez@espoch.edu.ec, ORCID: 0000-0002-9255-3168

${ }^{4}$ Escuela Superior Politécnica de Chimborazo, Facultad de Mecánica Riobamba, Ecuador. luis.choto@espoch.edu.ec, ORCID 0000-0003-2499-3337
} 
thicknesses schedule 40, XS and 80, in addition, two cracks are introduced in the surface of the welded joint of 2 and $4 \mathrm{~mm}$ in length, the geometric model was made using Solidworks software and the static analysis with finite elements was carried out using ANSYS software, the results were analyzed in SPSS with the multiple linear regression method. Results. The independent variable with the greatest influence on the dependent one is the diameter of the pipe, followed by the length of the crack and finally the thickness of the pipes, this measured by the correlation between the variables and the stepwise regression model. Conclusion: All the variables have a linear behavior and directly proportional to the allowed force applicable to the welded joint.

Keywords: numerical simulation, welding, cracks, pipe.

\section{Resumen}

Introducción. Las fisuras son las discontinuidades más críticas que se presentan en la soldadura, desplegando una alta concentración de esfuerzos y por ende una reducción de las cargas que se pueden aplicar a las juntas soldadas, normas de construcción de sistemas de transportación de oleoductos como la API 1104, es muy estricta respecto a la aceptación de estas discontinuidades, pero con una fundamentación empírica. Objetivo. Determinar la influencia que tiene la longitud de las fisuras en el esfuerzo de fluencia de juntas soldadas con el acero ASTM A36 mediante simulación numérica por elementos finitos. Metodología. Se realiza un análisis cualitativo de determinación de la carga que se puede aplicar a tuberías unidas mediante soldadura con fisuras, las variables independientes son el espesor de tubería, la longitud de la fisura y el diámetro de tubería. Se selecciona tuberías comerciales desde 4 hasta 20 in de diámetro nominal, con 3 espesores cedula 40, XS y 80, además se introduce dos fisuras en la superficie de la junta soldada de 2 y $4 \mathrm{~mm}$ de longitud, el modelo geométrico se realizó en el software Solidworks y el análisis estático con elementos finitos en ANSYS, los resultados fueron analizados en SPSS mediante el método de regresión lineal múltiple. Resultados. La variable independiente con mayor influencia en la dependiente es el diámetro de la tubería, seguida de la longitud de la fisura y finalmente el espesor de las tuberías, esto medida por la correlación entre las variables y el modelo a pasos de la regresión. Conclusión. Todas las variables tienen un comportamiento lineal y directamente proporcional a la fuerza permitida aplicable a la junta soldada

Palabras claves: Simulación numérica, soldadura, fisuras, tubería

\section{Introducción}

Actualmente la simulación numérica se ha convertido en una prioridad tanto en temas de investigación como en aplicaciones industriales, dando mejor eficiencia y eficacia al desarrollo de nuevos productos ingenieriles y a su vez prediciendo eventos físicos que se pueden desarrollar a la hora de la utilización de un producto diseñado bajo normas o parámetros con la ayuda de la simulación. 
Un estudio doctoral denominado "Análisis de mecánica de fractura en tuberías soldadas de gaseoducto mediante simulación numérica por elementos finitos" (Yépez, 2015), hace un estudio minucioso del comportamiento de la tubería con dos tipos de parámetros, tubería con fisura superficial semi elíptica en dirección axial bajo presión interna y tubería con fisura superficial semi elíptica en dirección transversal bajo carga axial. Los resultados obtenidos tanto en ANSYS 14.5 como los obtenidos por métodos analíticos (API 579, 2007), según la norma de los FTI (Factor de Intensidad de Tensiones) que es la tenacidad a la fractura de un material con una fisura presenta un error menor al $10 \%$ en ambos casos de la ubicación de la fisura de acuerdo con el eje longitudinal y transversal de la carga aplicada. Estos resultados comprueban una factibilidad de un análisis de la mecánica de la fractura elástico lineal.

En Pozo et al. (2017), se hace referencia a una "simulación numérica de la presencia de una grieta cercana a la soldadura de una unión tubular en T", en donde evalúa el desplazamiento tanto de la unión soldada como la apertura de la fisura mediante simulación numérica y comparando con datos experimentales.

Los resultados obtenidos en la investigación muestran una similitud de la simulación con los datos experimentales realizadas por Zerbst et al. (2002), en tanto al desplazamiento de la junta soldada con un $12 \%$ de diferencia para el comportamiento no lineal. Este análisis sirvió para la comparación de desplazamientos del cordón de soldadura y apertura de una grieta semi elíptica, y una validación de la simulación numérica sin obtener un esfuerzo a tracción de la soldadura.

Otra de las investigaciones denominado "Investigation on size tolerance of pore defect of girth weld pipe" se enfoca en medir la resistencia a la tracción y compresión de una tubería con un poro en el cordón de soldadura, el poro ha sido ubicado en tres posiciones, próximo a la superficie externa de la soldadura, en el centro del cordón de soldadura y en cerca de la pared interna del cordón de soldadura en dos materiales distintos API 5L X80, APY 5L X90, obteniendo resultados del esfuerzo de Von Mises que se concentran en el defecto poro, sin tener mayor influencia de la ubicación del mismo, sino más bien en la forma de dicho poro (Li, 2018).

El trabajo realizado por Massa \& Giudici (2009), denominado "Comportamiento de un gasoducto con fisuras", aborda la seguridad e integridad de las tuberías que provee de gas natural a la ciudad de Córdova (Argentina) que ha operado por más de 40 años. Se enfoca en cuantificar el estado tensional con modelos basados en determinar la tenacidad del material. Los resultados obtenidos muestran cuando serán críticos los tamaños de las fisuras al operar con una presión de servicio, el tamaño de las fisuras a la cual son tolerables según un factor de seguridad y finalmente la profundidad mínima que debe tener un eventual defecto para poder garantizar no produzca una falla.

La simulación numérica toma impulso en observar el comportamiento de materiales metálicos (Suarez, s.f.), evalúa el comportamiento a tensión de un alambre de acero perlítico con falta de cohesión producto del crecimiento de micro huecos en la solidificación del material. El trabajo presenta un modelo de falla hasta la rotura con una 
comparación experimental y simulación en el software ABACUS y una subrutina UEL. Esto abre las puertas a investigaciones relacionadas, al existir una similitud entre los resultados obtenidos mediante laboratorio y mediante software.

Si bien es cierto existen trabajos relacionados al tema en estudio con un enfoque de mecánica de la fractura, en el trabajo realizado por Yépez (2015), se enfoca en la determinación de la tenacidad a la fractura sin análisis de esfuerzos y para tuberías de transportación de gas. En Pozo et al. (2017), se realiza una simulación con una fisura en una soldadura tipo $\mathrm{T}$ de tuberías, pero sin mayor cambio de parámetros, y la investigación realizada por Li (2018), enfoca si a la determinación del esfuerzo de una tubería con poros con la variación de forma y ubicación de los mismo, este último trabajo es el que más se asemeja al caso de estudio propuesto.

De acuerdo con esto se plantea ubicar una fisura en un cordón de soldadura para tuberías de transportación de oleoductos y la aportación del trabajo es evaluar el esfuerzo de Von Mises con la diversificación de varios parámetros como diámetro de tuberías, espesor de tuberías, y dimensión de la fisura en el cordón. Los parámetros tanto de diámetro y espesor de tuberías se seleccionarán de acuerdo con los utilizados en la industria petrolera, mientras que la variación de resto de parámetros (dimensión de fisuras) se hará de acuerdo con criterios empíricos ya que no se puede controlar estos parámetros en la práctica real y será una aproximación a la realidad. Para determinar correlaciones y parámetros de mayor influencia en la resistencia a la tracción de Von Mises se utilizarán técnicas multivariantes con la ayuda del software SPSS.

\section{Metodología}

El procedimiento debe ser lógico y secuencial, iniciando con la elaboración de un modelo geométrico y selección de parámetros de medida, para luego iniciar un proceso de simulación en ANSYS a fin de recopilar los datos para un futuro análisis según una regresión lineal múltiple. El proceso inicia con la selección de diámetros y espesores de tubería, lo cual se considera medidas que se comercialicen para un análisis real, la unión de estas tuberías se realiza mediante soldadura por lo que también se prepara un modelo de la unión junto con las fisuras de dos longitudes distintas. La simulación se realiza mediante un proceso con parámetros específicos a fin de obtener resultados reales, para posteriormente analizar los resultados.

\section{Modelo geométrico de la junta}

En base a la Norma API 1104, para el análisis se tomarán diámetros y espesores según catálogos de fabricantes, para obtener resultados reales con la utilización de dimensiones de tuberías comerciales que se apeguen a la Norma analizada.

Se eligen tres espesores de tuberías, cedula 40, cedula XS y cedula 80, también es importante hacer una selección de diámetros y espesores de acuerdo con la preparación que se le debe hacer al material antes de la soldadura (bisel) ya que el tipo de unión es a tope. Según la Norma AWS (2002), para realizar un bisel en una soldadura a tope se 
necesita un espesor mínimo de $6 \mathrm{~mm},(1 / 4 \mathrm{in})$, de acuerdo con esto se elige diámetros y espesores que cumplan este requerimiento como se muestra en la tabla 1.

Tabla 1

Diámetros y espesores de tuberías comerciales según API 5L GRADO B

\begin{tabular}{|c|c|c|c|c|}
\hline DIÁMETRO & DIÁMETRO & ESPESOR & ESPESOR & ESPESOR \\
\hline NOMINAL & EXTERIOR & CÉDULA & CÉDULA & CÉDULA \\
\hline (in) & $(\mathrm{mm})$ & $40(\mathrm{~mm})$ & $\mathrm{XS}(\mathrm{mm})$ & $80(\mathrm{~mm})$ \\
\hline 4 & 114.3 & 6.02 & 8.56 & 8.56 \\
\hline 6 & 168.3 & 7.11 & 10.97 & 10.97 \\
\hline 8 & 219.1 & 8.18 & 12.7 & 15.09 \\
\hline 10 & 273.0 & 9.27 & 12.7 & 15.09 \\
\hline 12 & 323.8 & 10.31 & 12.7 & 17.48 \\
\hline 14 & 355.6 & 11.13 & 12.7 & 19.05 \\
\hline 16 & 406.4 & 12.7 & 12.7 & 21.44 \\
\hline 18 & 457 & 14.27 & 12.7 & 23.83 \\
\hline 20 & 508 & 15.9 & 12.7 & 26.19 \\
\hline
\end{tabular}

Fuente: Fiorella Representaciones S.A. (2018)

Dimensionamiento del cordón de soldadura

Las dimensiones del cordón de soldadura son estándar, de acuerdo con el WPS (Welding Procedure Specification) en concordancia a la Norma API 1104, como se indica en la Figura 1. Esta norma brinda las especificaciones técnicas de los procesos de soldadura para uniones de tuberías para la transportación de oleoductos.

\section{Figura 1}

Dimensiones del cordón de soldadura a tope con Bisel tipo V

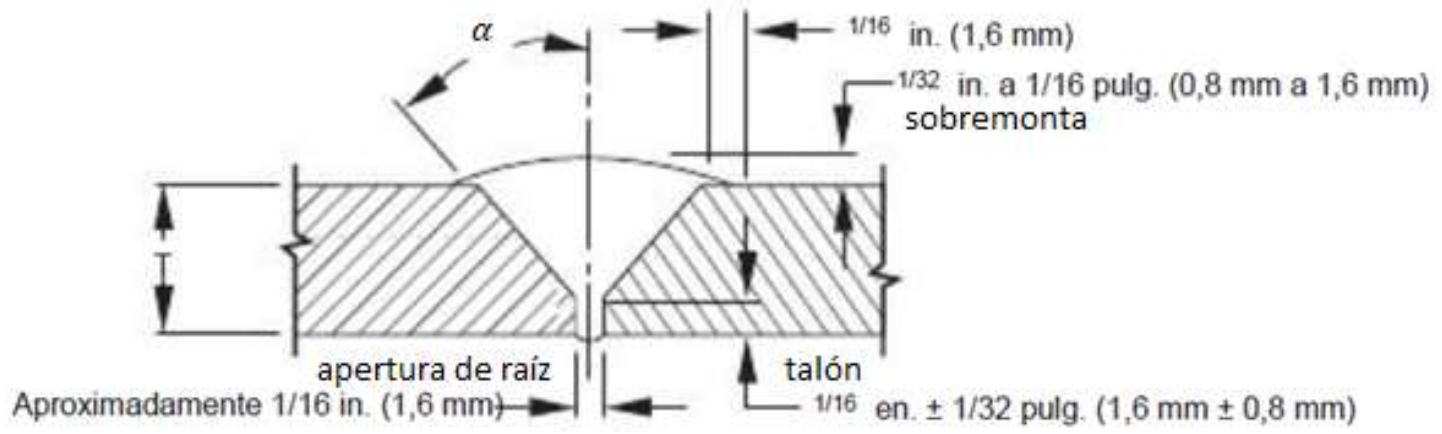

Fuente: API 1104 (2013)

Como existe un rango que puede variar cada una de estas dimensiones se asigna un valor constante a cada una de ellas: 


\section{Tabla 2}

Dimensiones del cordón de soldadura

\begin{tabular}{ll}
\hline ESPECIFICACIÓN & DIMENSIÓN \\
\hline Apertura de raíz & $1,6(\mathrm{~mm})$ \\
Altura de raíz (talón) & $1,6(\mathrm{~mm})$ \\
$\begin{array}{l}\text { Refuerzo de la soldadura (Sobre } \\
\text { monta) }\end{array}$ & $1,6(\mathrm{~mm})$ \\
$\alpha$ (ángulo de apertura - bisel V) & $30^{\circ}$ \\
\hline
\end{tabular}

Fuente: API $1104(2013)$

Con estos datos se formula un modelo geométrico para el análisis de la simulación en el software Solid Works, esto se lo realiza con diámetros y espesores dados en la tabla 1, además se utiliza las medidas del cordón de soldadura según la tabla 2.

\section{Figura 2}

Modelo geométrico de una tubería soldada de diámetro 168,3 mm y espesor

\section{7,11 $\mathrm{mm}$ sin fisuras}

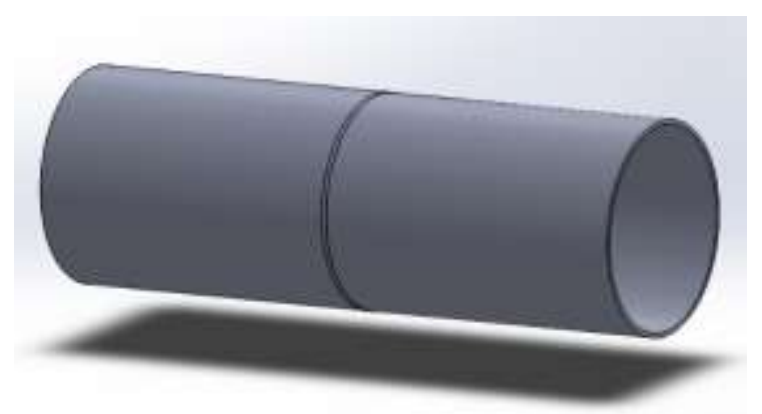

Fuente: Elaboración propia.

Análisis y dimensiones de las fisuras

Uno de los parámetros más importantes para el análisis de esfuerzos es la forma y dimensiones de las fisuras en el cordón de soldadura. Es lógico que en una fisura existe una concentración de tensiones lo cual debilitara al material, disminuyendo la carga que se puede aplicar, un caso típico es el análisis de fisuras de forma elíptica aproximándose a la realidad, que muestra las tensiones máximas en el borde del hueco. Esto hará que se pueda analizar en la simulación que realmente el esfuerzo máximo se concentra en el borde de dicha fisura como se muestra en la figura 3 (Oller, 2001). 


\section{Figura 3}

Placa finita con un orificio elíptico, sometida a un estado de tensiones

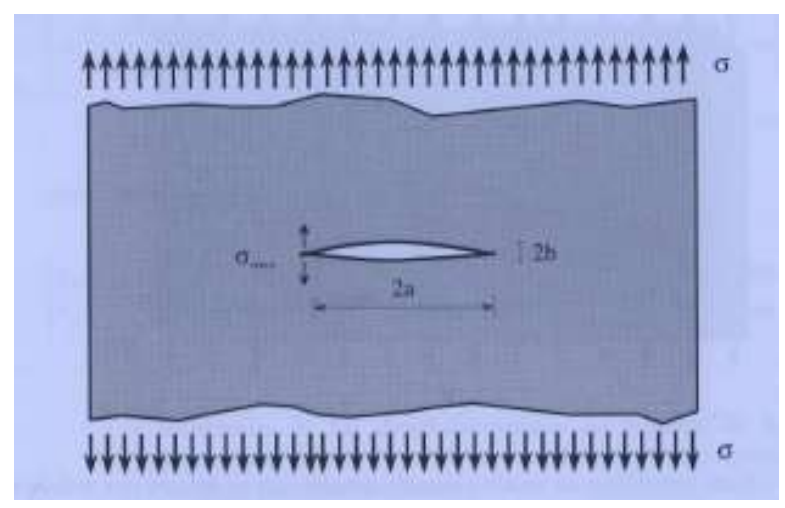

Fuente: Oller (2001)

Para ello se plantea diferentes dimensiones de las fisuras, considerando:

$$
\frac{a}{b}>>0,5
$$

Esto en base al modelo planteado por Inglis, para definir el estado de tensiones máximos en el borde de la elipse (Jayatilaka, 1979).

Con esta fundamentación se plantea fisuras elípticas con dimensiones para cada combinación de diámetro y espesor de tubería como se muestra en la tabla 3.

\section{Tabla 3}

Dimensiones de las fisuras para el análisis

\begin{tabular}{llll}
\hline & $2 \mathrm{a}(\mathrm{mm})$ & $2 \mathrm{~b}(\mathrm{~mm})$ & $\begin{array}{l}\text { Profundidad } \\
(\mathrm{mm})\end{array}$ \\
\hline FISURA 1 & 2 & 0,5 & 1 \\
FISURA 2 & 4 & 0,5 & 1
\end{tabular}

Fuente: Elaboración propia

Nótese que la relación $\frac{a}{b}$ siempre es mayor a 0,5 y la profundidad $1 \mathrm{~mm}$, esto para determinar cuál es la influencia de la longitud de una fisura en el esfuerzo a tensión.

Con esto se define una cantidad de 54 simulaciones para un posterior análisis en SPSS.

Es importante también mencionar que las fisuras estarán ubicadas en la parte externa del cordón de soldadura a fin de reducir el tamaño de la muestra. 


\section{Figura 4}

Geometría de la fisura en el cordón de soldadura

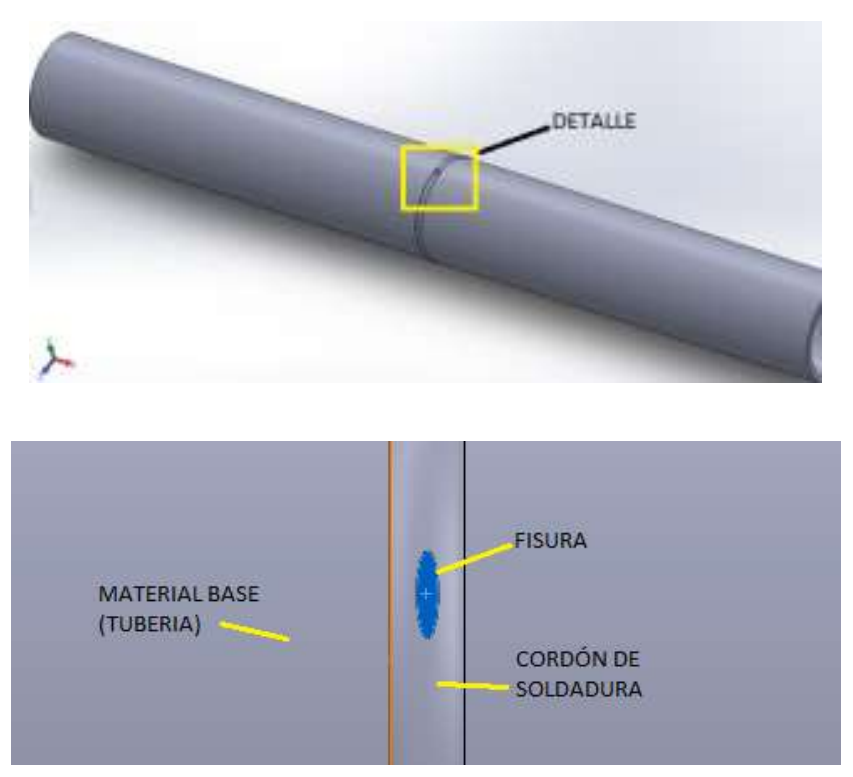

Fuente: Elaboración propia.

\section{Definición de malla}

Se debe aplicar elementos sólidos como tetraedro de 10 nodos y elementos de ladrillo de 20 nodos para el análisis de tensión (Chen, 2014). Para este estudio se escoge elementos tetraédricos con 10 nodos para el mallado general en el cordón de soldadura.

\section{Figura 5}

Mallado brusco

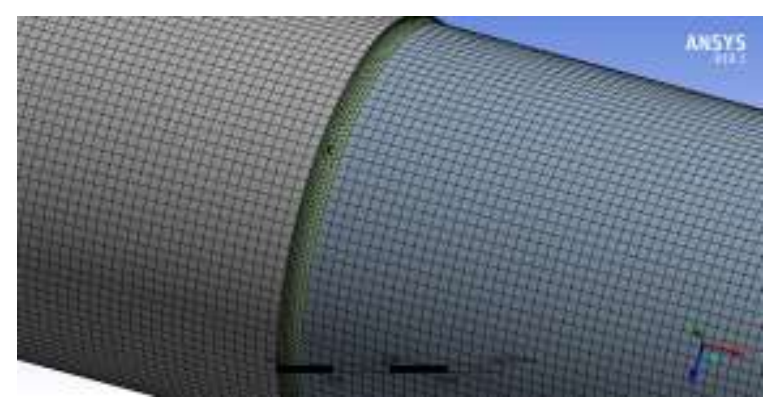

Fuente: Elaboración propia.

Para mejorar los resultados es necesario mejorar la malla, optando por utilizar un tamaño de elemento de $3 \mathrm{~mm}$ para la tubería y de 1,5 mm para el cordón de soldadura, como se requiere el análisis de esfuerzos en la fisura se debe mejorar mucho más en dicha ubicación, mediante un mallado local, creando una esfera con elemento de 0,25 mm. 


\section{Figura 6}

Mallado local en la fisura

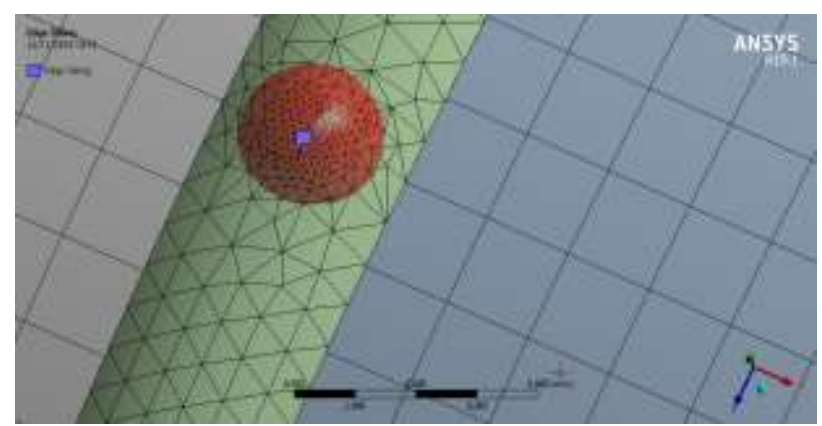

Fuente: Elaboración propia.

Ahora se debe comprobar si la malla es adecuada, mediante la utilización de Element Quality.

La opción de calidad del elemento proporciona una métrica de calidad compuesta que varía entre 0 y 1 . Esta métrica se basa en la relación del volumen con la suma del cuadrado de las longitudes de los bordes para elementos 2D quad / tri, o la raíz cuadrada del cubo de la suma del cuadrado de las longitudes de borde para elementos $3 \mathrm{D}$. Un valor de 1 indica un cubo o cuadrado perfecto, mientras que un valor de 0 indica que el elemento tiene un volumen cero o negativo (ANSYS, 2019).

Para 3D se puede expresar como:

$$
\text { Quality }=C\left[\frac{\text { volume }}{\sqrt{\left[\sum(\text { Edge Length })^{2}\right]^{3}}}\right]
$$

El valor de C depende del tipo de elemento, según la tabla 4.

\section{Tabla 4}

Valor de la constante C para determinar la calidad de malla

\begin{tabular}{ll}
\hline Element & Value of C \\
\hline Triangle & 6.92820323 \\
Quadrangle & 4.0 \\
Tetrahedron & 124.70765802 \\
Hexagon & 41.56921938 \\
Wedge & 62.35382905 \\
Pyramid & 96
\end{tabular}

Fuente: ANSYS (2019)

De manera más práctica, el software hace un cálculo interno con la formula dada anteriormente y muestra un código de colores, azul destaca como una malla perfecta a lo contrario del rojo (ANSYS RELEASE, 2010). 


\section{Figura 7}

\section{Código de colores para calidad de malla}

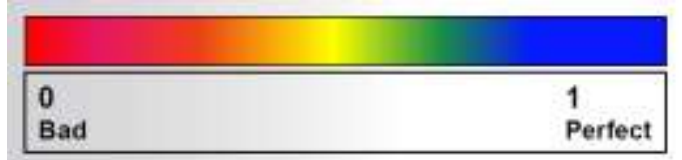

Fuente: ANSYS RELEASE (2010)

Con el fundamento dado, se compara el modelo mallado y se determina si es correcto la malla asignada.

\section{Figura 8}

Código de colores para calidad de malla en la fisura

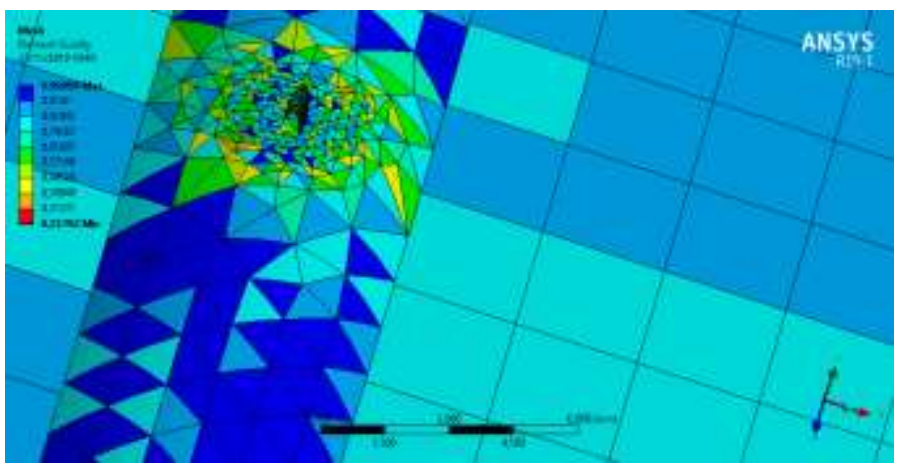

Fuente: Elaboración propia.

El valor máximo es 0,99 y el valor mínimo 0,22 , en la fisura que es el interés de mallado y por ende de determinación del esfuerzo se tiene un valor mínimo de 0,48.

\section{Modelo físico}

Un elemento mecánico al estar sometido a cargas axiales tiende a sufrir un estado de esfuerzos internos y una deformación total, esto se puede determinar mediante un ensayo denominado tracción uniaxial, que consiste en aplicar cargas variables a una probeta e ir determinando cuál es su deformación con un área transversal y longitud inicial conocida.

Cabe mencionar que todo diseño estructural se lo realiza hasta llegar el esfuerzo de fluencia del material $\sigma_{y}$ como máximo, esto debido a que es imposible tener una deformación plástica en el elemento estructural. La máxima carga que puede soportar un elemento estructural o un componente de maquinaria en condiciones normales de uso es considerablemente más pequeña que la carga de fluencia. Esta carga más pequeña se conoce como la carga permisible y, en ocasiones, como la carga de trabajo o carga de diseño. Así, sólo una fracción de la capacidad última de carga del elemento se utiliza cuando se aplica la carga permisible. El remanente de la capacidad portadora de carga del elemento se mantiene en reserva para asegurar su desempeño seguro. La razón de la carga de fluencia a la carga permisible se emplea para definir el factor de seguridad (Beer et al., 2012). Se tiene que: 


$$
F S=\frac{\text { Esfuerzo de fluencia }}{\text { Esfuerzo de trabajo }}
$$

Donde FS representa el factor de seguridad que siempre deberá ser un valor mayor a 1 y como mínimo de 1.

Con este fundamento se define el fenómeno físico de la tubería con carga uniaxial de tracción, en un borde como fijo y el otro borde con la aplicación de una carga constante (PRESIÓN DE $250 \mathrm{MPa}=36259$ psi que es el límite de fluencia del material API 5L Grado B), si la conexión de la tubería no presenta ninguna fisura el mínimo factor de seguridad deberá ser de 1.

\section{Figura 9}

Tracción uniaxial de la tubería

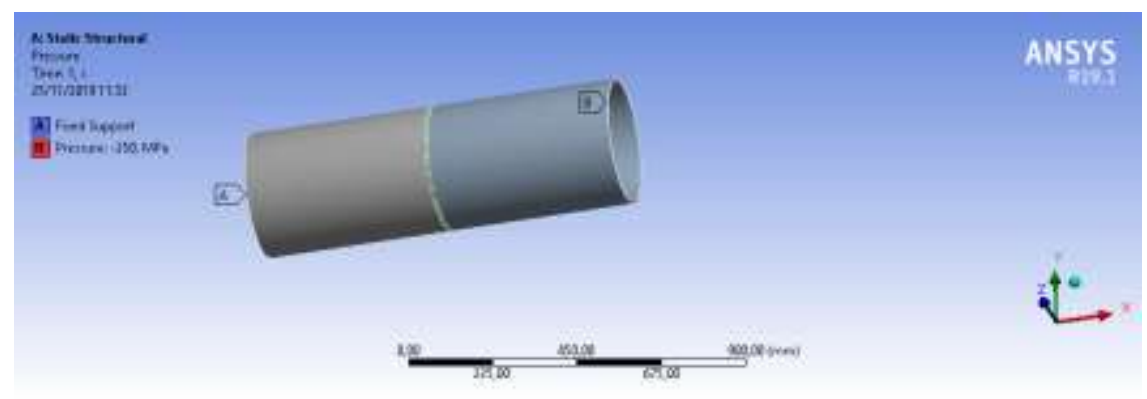

Fuente: Elaboración propia.

\section{Convergencia de resultados}

Es necesario, luego de una programación manual de mallado, refinar nuevamente el número de veces que sea necesario a fin de garantizar un resultado que converja hasta llegar a un error menor al $10 \%$ entre un resultado y otro. El cambio brusco de resultados se da por un mínimo cambio de malla, para evitar datos atípicos en la simulación se programa en ANSYS un refinamiento automático hasta que el resultado tenga una convergencia menor al $10 \%$.

Un ejemplo es el de la tubería de 4in. de diámetro nominal con la fisura 1, refinando 3 veces la malla a fin de que en el esfuerzo de Von Mises haya un cambio menor al $10 \%$. Como resultado se obtiene un cambio de apenas el $1,4589 \%$ del resultado y por ende representa un valor real.

\section{Tabla 5}

Reporte de ANSYS esfuerzo máximo y equivalente

\begin{tabular}{ccccc}
\hline & $\begin{array}{c}\text { Equivalent } \\
\text { Stress (MPa) }\end{array}$ & $\begin{array}{c}\text { Change } \\
(\%)\end{array}$ & Nodes & Elements \\
\hline 1 & 341,28 & & 467000 & 104815 \\
2 & 448,97 & 27,255 & 484314 & 116810 \\
3 & 639,26 & 34,972 & 559597 & 171309 \\
4 & 648,65 & 1,4589 & 767408 & 322897 \\
\hline
\end{tabular}




\section{Figura 10}

Convergencia de resultados del esfuerzo máximo en la fisura 1

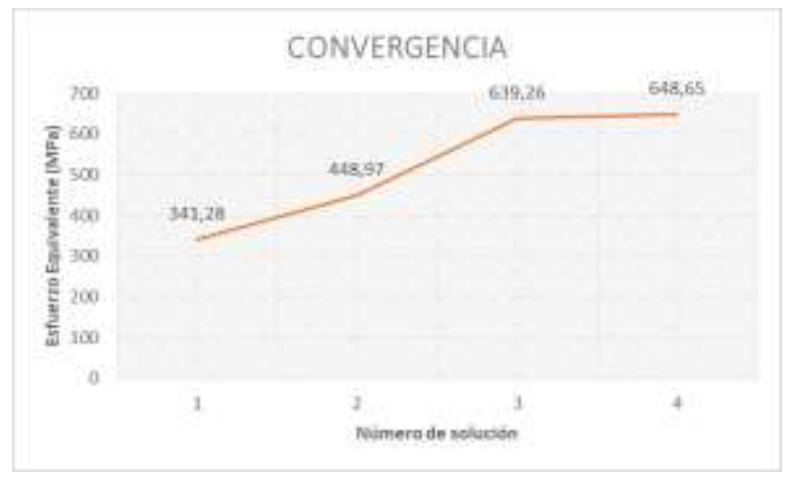

Fuente: Elaboración propia

Para el análisis de esfuerzos en la fisura se describió el proceso mediante la ecuación:

$$
\sigma=\sigma_{V M}=\frac{1}{\sqrt{2}} \sqrt{\left(\sigma_{1}-\sigma_{2}\right)^{2}+\left(\sigma_{2}-\sigma_{3}\right)^{2}+\left(\sigma_{3}-\sigma_{1}\right)^{2}}
$$

Lógicamente en la fisura se concentran esfuerzos, mayorando al que debería soporta dicha soldadura. Además, se puede obtener el factor de seguridad FS después de la simulación.

\section{Figura 11}

Factor de seguridad mínimo en la fisura (ejemplo para tubería de 6 in. con la fisura 1)

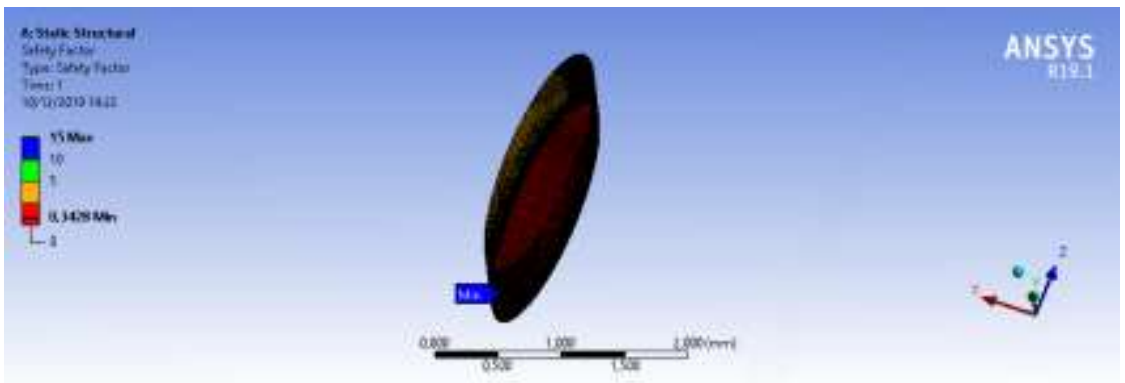

Fuente: Elaboración propia.

Figura 12

Esfuerzo de Von Mises máximo en la fisura (ejemplo para tubería de 6 in. con la fisura 1)

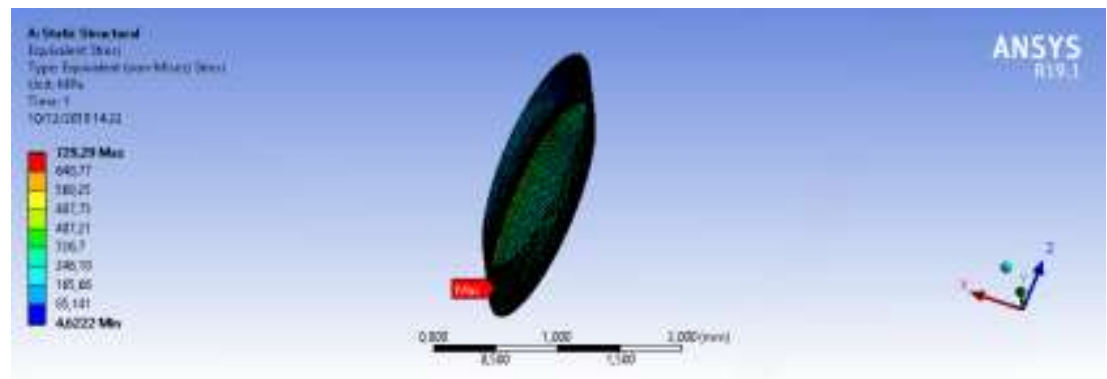

Fuente: Elaboración propia. 
En este análisis en particular como ejemplo se ha tomado una tubería con el cordón de soldadura incorporado una fisura obteniendo los siguientes resultados:

\section{Tabla 6}

Resultados obtenidos de esfuerzo y FS en la fisura

$\begin{array}{lllll}\text { Diámetro externo }(\mathrm{mm}) & \text { Espesor }(\mathrm{mm}) & \text { Fisura } & \sigma_{V M}(\mathrm{MPa}) & \mathrm{FS}(\text { Adimensional) }\end{array}$

\begin{tabular}{lllll}
\hline 168,3 & 7,11 & 1 & 729,29 & 0,3428 \\
\hline
\end{tabular}

Fuente: Elaboración propia.

\section{Resultados}

Prolongando como ejemplo con el caso anterior (tabla 6.), el esfuerzo máximo en la fisura presenta un valor de 729,29 MPa y un factor de seguridad de 0,3428, lo cual indica un riesgo en la junta soldada porque el material podría soportar solamente $250 \mathrm{MPa}$. Mediante una serie de pasos es posible determinar cuál es la reducción de la tensión que podría soportar la unión soldada. Si el FS es 0,3428 significa que para llegar a 1 faltaría 0,6572, con este valor se deberá reducir el 65,72\% de los $250 \mathrm{MPa}$. Es decir, el esfuerzo máximo que se debe someter a la junta soldada será de 85,7 MPa. Al cambiar las condiciones físicas con esta carga uniaxial en ANSYS el FS deberá mantenerse en 1 en el punto de máxima concentración de esfuerzos.

\section{Figura 13}

FS con la carga reducida

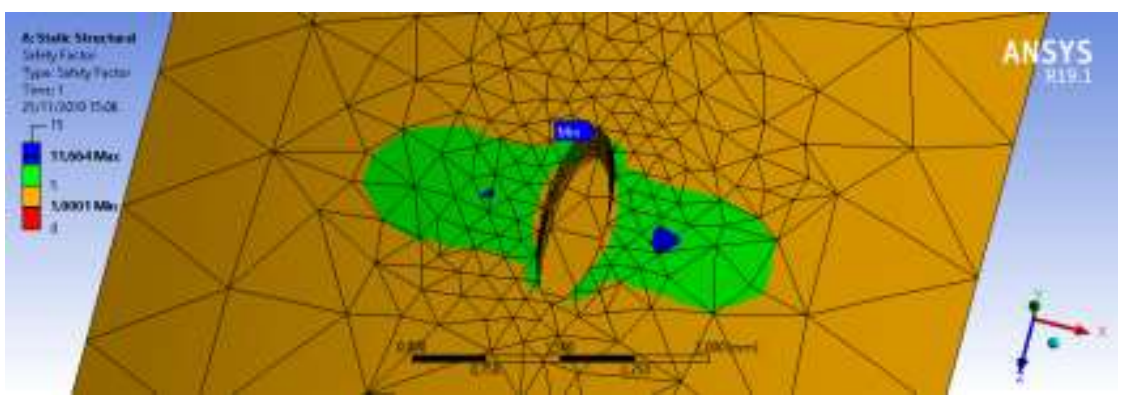

Fuente: Elaboración propia

Figura 14

Esfuerzo de Von Mises con la carga reducida

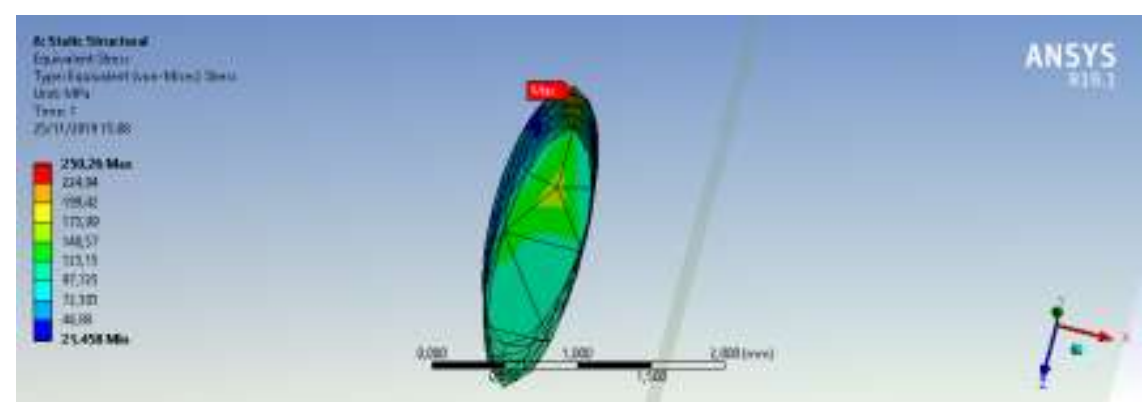

Fuente: Elaboración propia 
En las figuras 13. y 14. Se aprecia que el esfuerzo máximo en la fisura es de 250,26 MPa, lo que realmente debería soportar como máximo la junta soldada y el factor de seguridad de 1,0001 como mínimo, esto indica que la carga máxima a tracción que se puede aplicar a esta soldadura será de 85,7 MPa con una reducción del 65,72 \% del límite de fluencia del material base.

El área transversal de cada tubería es variable, y conociendo el esfuerzo máximo que se puede aplicar es posible determinar la fuerza a tracción que se puede aplicar al sistema de tuberías unidas con soldadura, para el ejemplo dado en este análisis se determina el esfuerzo que se puede aplicar para que la concentración de esfuerzos sea $250 \mathrm{MPa}$. en la fisura, es decir un valor de 85,7 MPa que se puede aplicar. Si se determina el área transversal $3600,45 \mathrm{~mm}^{2}$ para este ejemplo y determinando mediante la formula $\sigma=\frac{F}{A}$, la fuerza que se puede aplicar para que no falle el sistema es de 308559,1224 N.

Para cada diámetro de tubería se genera las seis simulaciones, con las dos longitudes de fisuras y tres espesores denominado cedulas, llevando un total de 54 simulaciones, con una variable dependiente (Fuerza Permitida) y tres variables independientes (Diámetro exterior de la tubería; espesor de la tubería y tipo de fisura ( $\left.\begin{array}{lll}1 & \text { o } & 2\end{array}\right)$ ). El análisis de resultados se realiza en SPSS.

De acuerdo con la tabla 7. de correlaciones entre la variable dependiente con las variables independientes no se podrían excluir ninguna variable, puesto que todas presentan una significación menor a 0,05 . La variable dependiente muestra correlaciones con valores de 0,$868 ; 0,800$ y $-0,321$ con las variables diámetro exterior, espesor y tipo de fisura respectivamente.

\section{Tabla 7}

Correlaciones entre variables

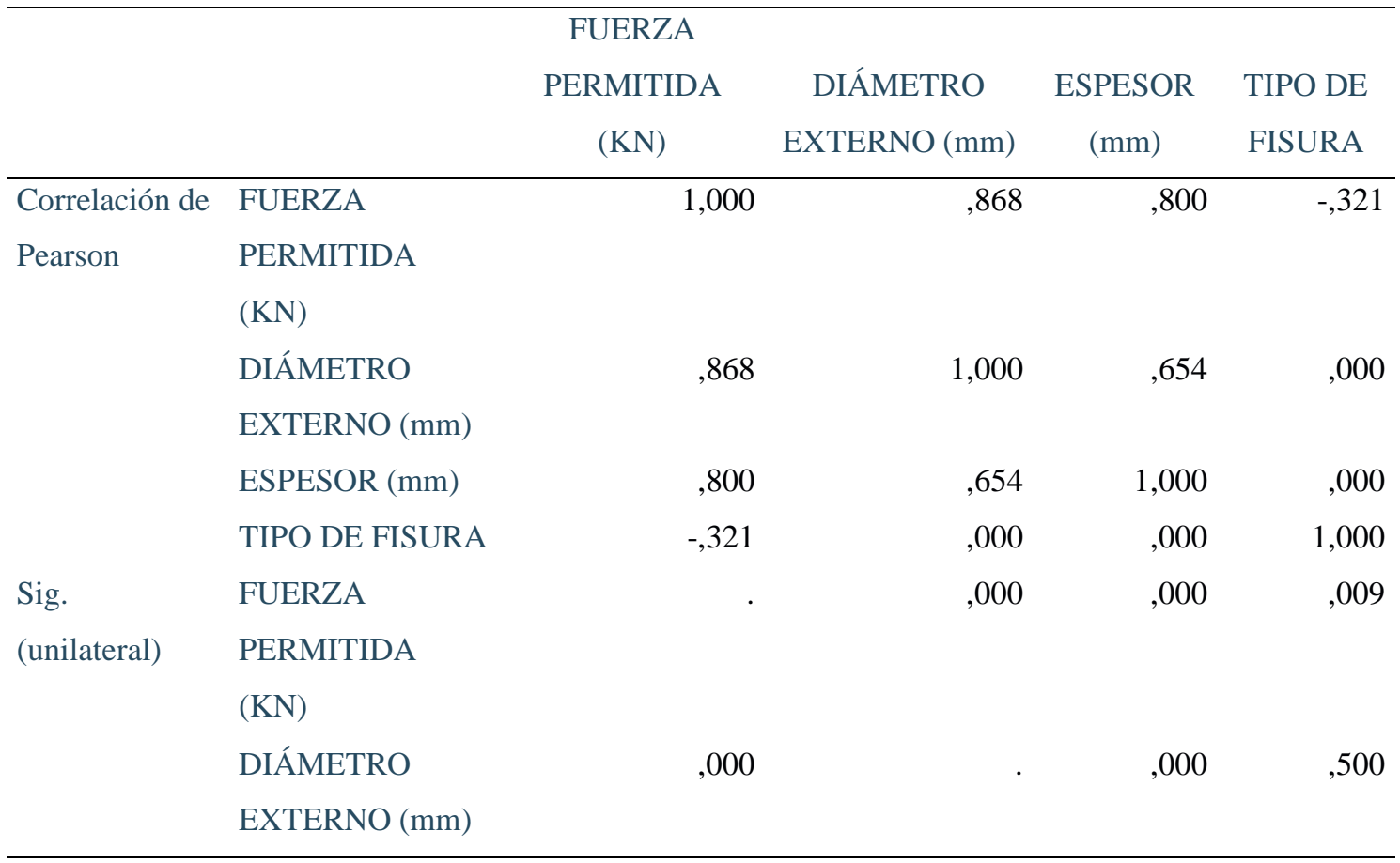


Vol. 4, N4.2, p. 77-100, diciembre, 2021

Tabla 7

Correlaciones entre variables (continuación)

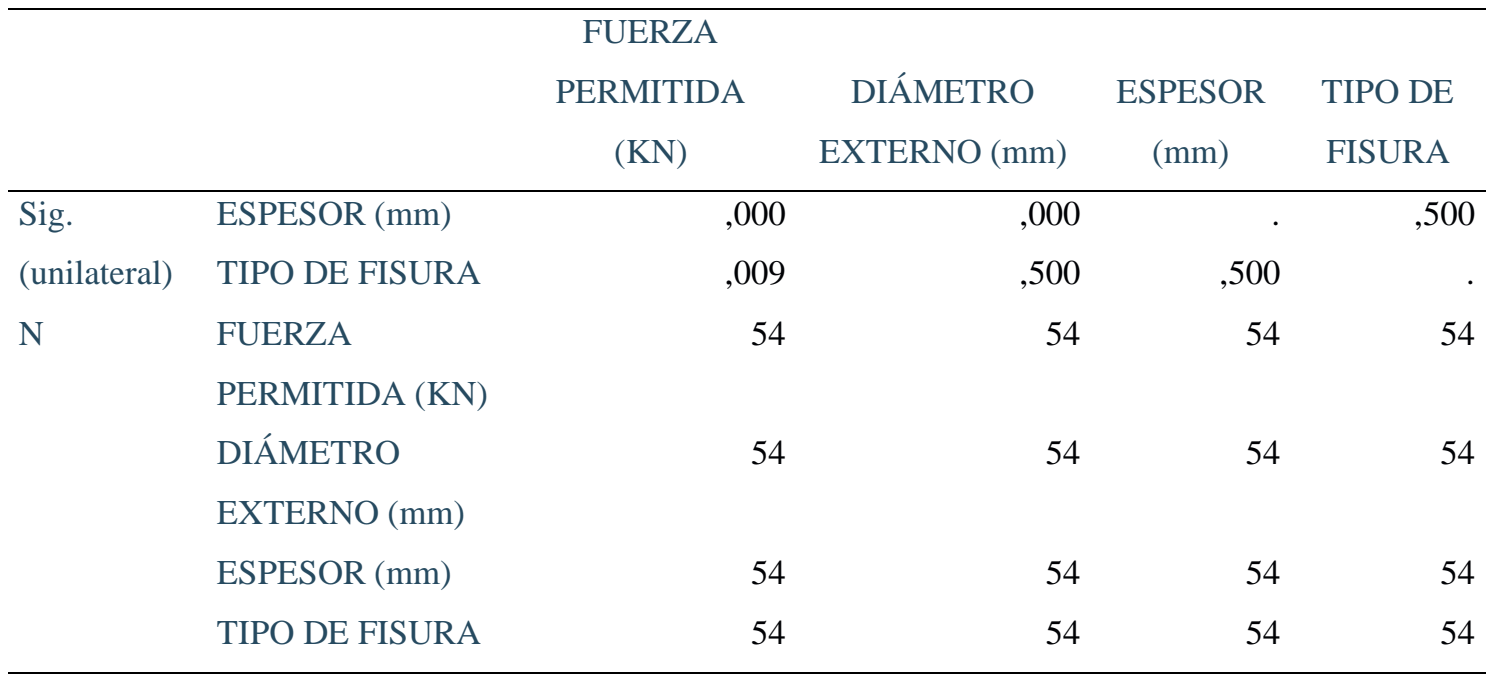

Fuente: Elaboración propia.

Como se utiliza un método a pasos, es posible fundamentar las variables que han entrado en cada modelo. En el primer modelo entra la variable Diámetro externo por tener mayor correlación, en el segundo modelo la variable tipo de fisura, y finalmente la variable espesor de la tubería. El método utiliza criterios de probabilidad de F, para entrar $<=0,050$ y Probabilidad de F para eliminar $>=, 100$ ). De acuerdo con la tabla 8. ninguna variable se elimina en los modelos propuestos.

Tabla 8

Variables entradas/salidas

\begin{tabular}{|c|c|c|c|}
\hline \multirow[b]{2}{*}{ Modelo } & \multicolumn{2}{|r|}{ Variables } & \multirow[b]{2}{*}{ Método } \\
\hline & Variables entradas & eliminadas & \\
\hline \multirow[t]{2}{*}{1} & DIÁMETRO & & . Por pasos (Criterios: Probabilidad-de-F-para-entrar $<=$ \\
\hline & EXTERNO (mm) & & ,050, Probabilidad-de-F-para-eliminar $>=, 100)$. \\
\hline \multirow[t]{2}{*}{2} & TIPO DE FISURA & & . Por pasos (Criterios: Probabilidad-de-F-para-entrar $<=$ \\
\hline & & & ,050, Probabilidad-de-F-para-eliminar $>=, 100)$ \\
\hline 3 & $\operatorname{ESPESOR}(\mathrm{mm})$ & & $\begin{array}{l}\text { Por pasos (Criterios: Probabilidad-de-F-para-entrar }<= \\
\text {,050, Probabilidad-de-F-para-eliminar }>=, 100) \text {. }\end{array}$ \\
\hline a. Varia & le dependiente: FUEF & PERMITID & $(\mathrm{KN})$ \\
\hline
\end{tabular}

Fuente: Elaboración propia

De acuerdo con la tabla 9. Es posible determinar la ecuación del modelo y la variación que puede tener cada coeficiente:

$\beta_{0}=14,183$, CONSTANTE

$\beta_{1}=1,846$

$\beta_{2}=-245.697$ 
$\beta_{3}=32.709$

$$
F P=14,183+1,846 * D E(m m)-245,697 * T F+32,709 * E(m m)
$$

Donde:

FP: es la fuerza permitida

DE: Diametro exterior en milimetros

TF: tipo de fisura 1,2

\section{E: Espesor en milimetros}

La fuerza permitida que se puede aplicar depende directamente del diámetro de tubería, espesor y de la longitud de la tubería. Esta fuerza a tracción se puede aplicar tal como se indica en la Figura 15.

\section{Figura 15}

Diagrama de representación de la Fuerza Permitida

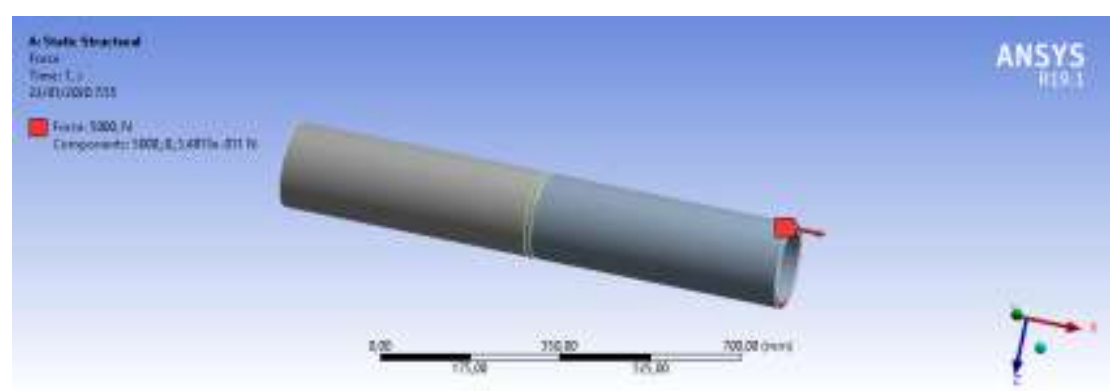

Fuente: Elaboración propia.

\section{Tabla 9}

Coeficientes de la regresión lineal múltiple

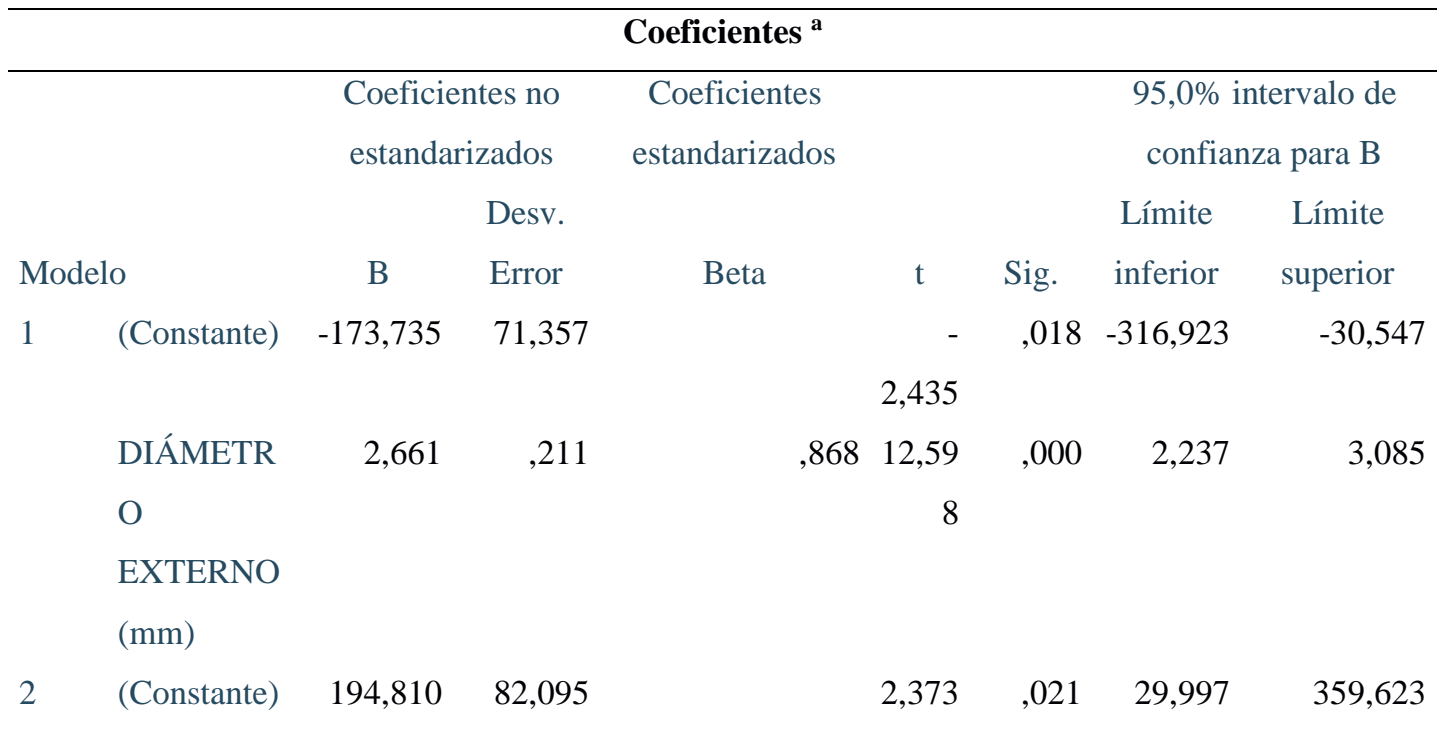




\section{Tabla 9}

Coeficientes de la regresión lineal múltiple (continuación)

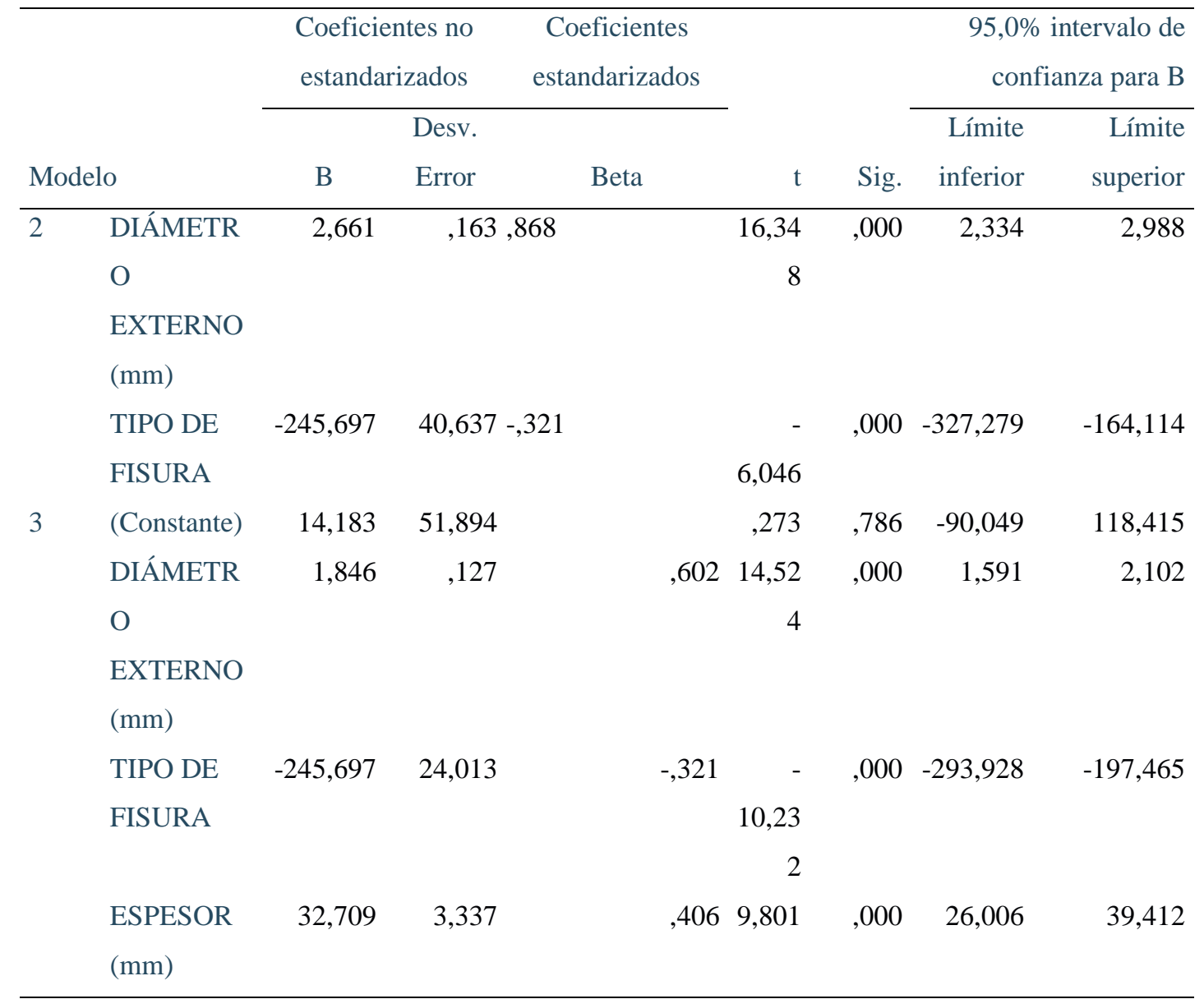

Fuente: Elaboración propia

La variación de los coeficientes estaría entre:

$\beta_{0} \in[-90.049,118.415]$

$\beta_{1} \in[1.591,2.102]$

$\beta_{2} \in[-293.928,-197.465]$

$\beta_{3} \in[26.006,39.412]$

Además, el modelo explica un 95,1\% de la varianza, según la Tabla 10.

\section{Tabla 10}

Resumen del modelo

\begin{tabular}{|c|c|c|c|c|c|c|c|c|c|c|}
\hline \multirow[b]{4}{*}{ Modelo } & \multirow[b]{4}{*}{$\mathrm{R}$} & \multirow{4}{*}{$\begin{array}{c}\mathrm{R} \\
\text { cuadrado }\end{array}$} & \multirow{4}{*}{$\begin{array}{c}\text { R } \\
\text { cuadrado } \\
\text { ajustado }\end{array}$} & \multicolumn{2}{|l|}{ Error } & \multicolumn{5}{|c|}{ Estadísticos de cambio } \\
\hline & & & & estándar de & Cambio & & & & Sig. & \\
\hline & & & & la & en $\mathrm{R}$ & Cambio & & & Cambio & Durbin- \\
\hline & & & & estimación & cuadrado & en $\mathrm{F}$ & $\mathrm{gl1}$ & $\mathrm{gl} 2$ & en $\mathrm{F}$ & Watson \\
\hline 1 &, $868^{\mathrm{a}}$ & ,753 & ,748 & 193,74448 & ,753 & 158,719 & 1 & 52 & ,000 & \\
\hline
\end{tabular}


Vol. 4, N4.2, p. 77-100, diciembre, 2021

\section{Tabla 10}

Resumen del modelo (continuación)

\begin{tabular}{|c|c|c|c|c|c|c|c|c|c|c|}
\hline \multirow[b]{4}{*}{ Modelo } & \multirow[b]{4}{*}{$\mathrm{R}$} & \multirow{4}{*}{$\begin{array}{c}\mathrm{R} \\
\text { cuadrado }\end{array}$} & \multirow{4}{*}{$\begin{array}{c}\text { R } \\
\text { cuadrado } \\
\text { ajustado }\end{array}$} & \multirow{4}{*}{$\begin{array}{c}\text { Error } \\
\text { estándar de } \\
\text { la } \\
\text { estimación }\end{array}$} & \multicolumn{5}{|c|}{ Estadísticos de cambio } & \multirow{4}{*}{$\begin{array}{l}\text { Durbin- } \\
\text { Watson }\end{array}$} \\
\hline & & & & & Cambio & & & & Sig. & \\
\hline & & & & & en $\mathrm{R}$ & Cambio & & & Cambio & \\
\hline & & & & & cuadrado & en $\mathrm{F}$ & gl1 & $\mathrm{gl} 2$ & en $\mathrm{F}$ & \\
\hline 2 &, $925^{\mathrm{b}}$ & ,856 &, 851 & 149,31001 &, 103 & 36,556 & 1 & 51 & ,000 & \\
\hline 3 &, $975^{\mathrm{c}}$ & ,951 & ,948 & 88,22897 & ,095 & 96,058 & 1 & 50 &, 000 & 2,760 \\
\hline \multicolumn{11}{|c|}{ a. Predictores: (Constante), DIÁMETRO EXTERNO (mm) } \\
\hline \multicolumn{11}{|c|}{ b. Predictores: (Constante), DIÁMETRO EXTERNO (mm), TIPO DE FISURA } \\
\hline \multicolumn{11}{|c|}{ c. Predictores: (Constante), DIÁMETRO EXTERNO (mm), TIPO DE FISURA, ESPESOR (mm) } \\
\hline \multicolumn{11}{|c|}{ d. Variable dependiente: FUERZA PERMITIDA (KN) } \\
\hline
\end{tabular}

Fuente: Elaboración propia

La correlación entre la variable dependiente (Fuerza Permitida $(\mathrm{KN})$ ) con la variable independiente (Diámetro exterior) muestra un valor cercano a 1 (0.868), además presenta un comportamiento lineal, la Figura 16. Indica que a medida que el diámetro aumenta la fuerza permitida a tracción que se puede aplicar sin que falle el material también tiende a crecer. Es lógico que las fisuras tipo 1 (longitud de $2 \mathrm{~mm}$.) de color azul tienden a ser menos peligrosas, por tanto, se puede aplicar una fuerza mayor, a lo contrario de las fisuras de $4 \mathrm{~mm}$. de longitud (color rojo), la fuerza que permiten es menor, por tanto, una fisura de mayor longitud tiende a aumentar la concentración de esfuerzos y por ende presenta mayor riesgo en un sistema de tuberías. Cabe aclarar que existen 6 valores con el mismo diámetro, es porque se utiliza un mismo diámetro para realizar 6 simulaciones con 3 diferentes espesores y 2 longitudes de tubería.

Figura 16

Dispersión de Fuerza Permitida vs Diámetro Externo

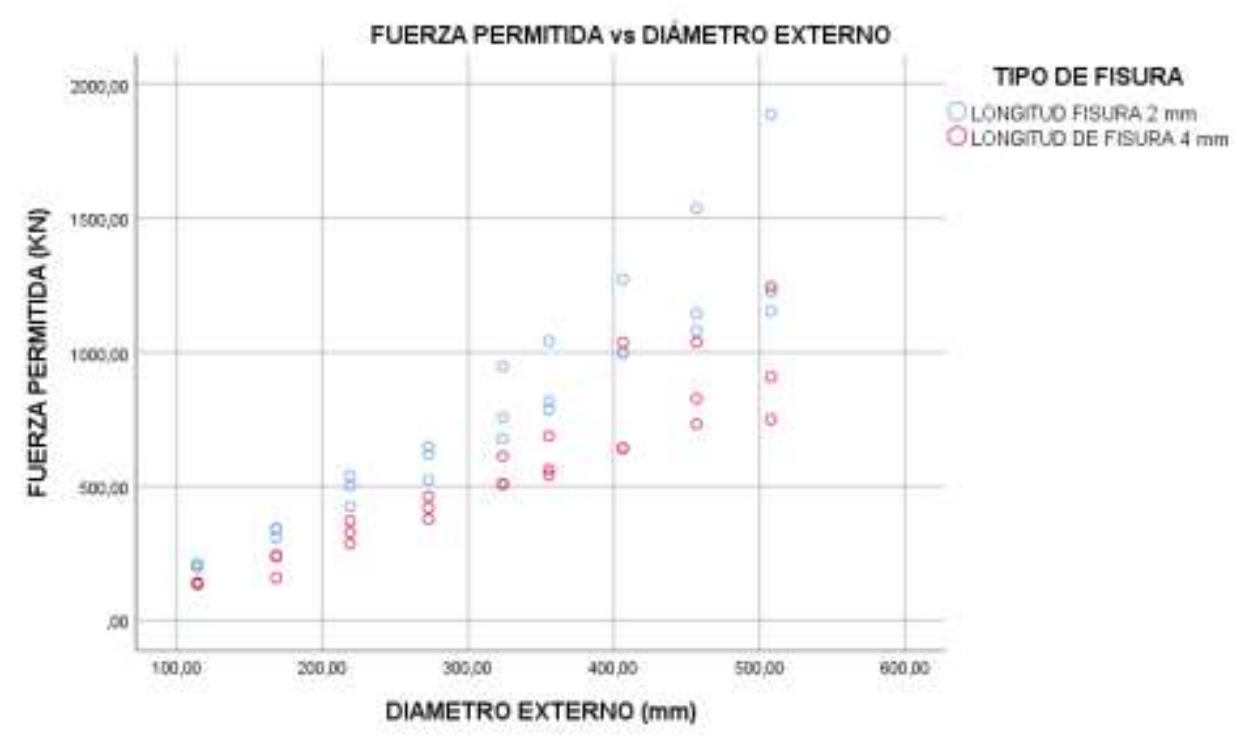

Fuente: Elaboración propia 
El mismo procedimiento se lo realiza para analizar la variable dependiente vs espesor de la tubería siempre y cuando se pueda contrastar el tipo de fisura que engloba la longitud de la discontinuidad.

\section{Figura 17}

Dispersión de fuerza permitida vs espesor de tubería

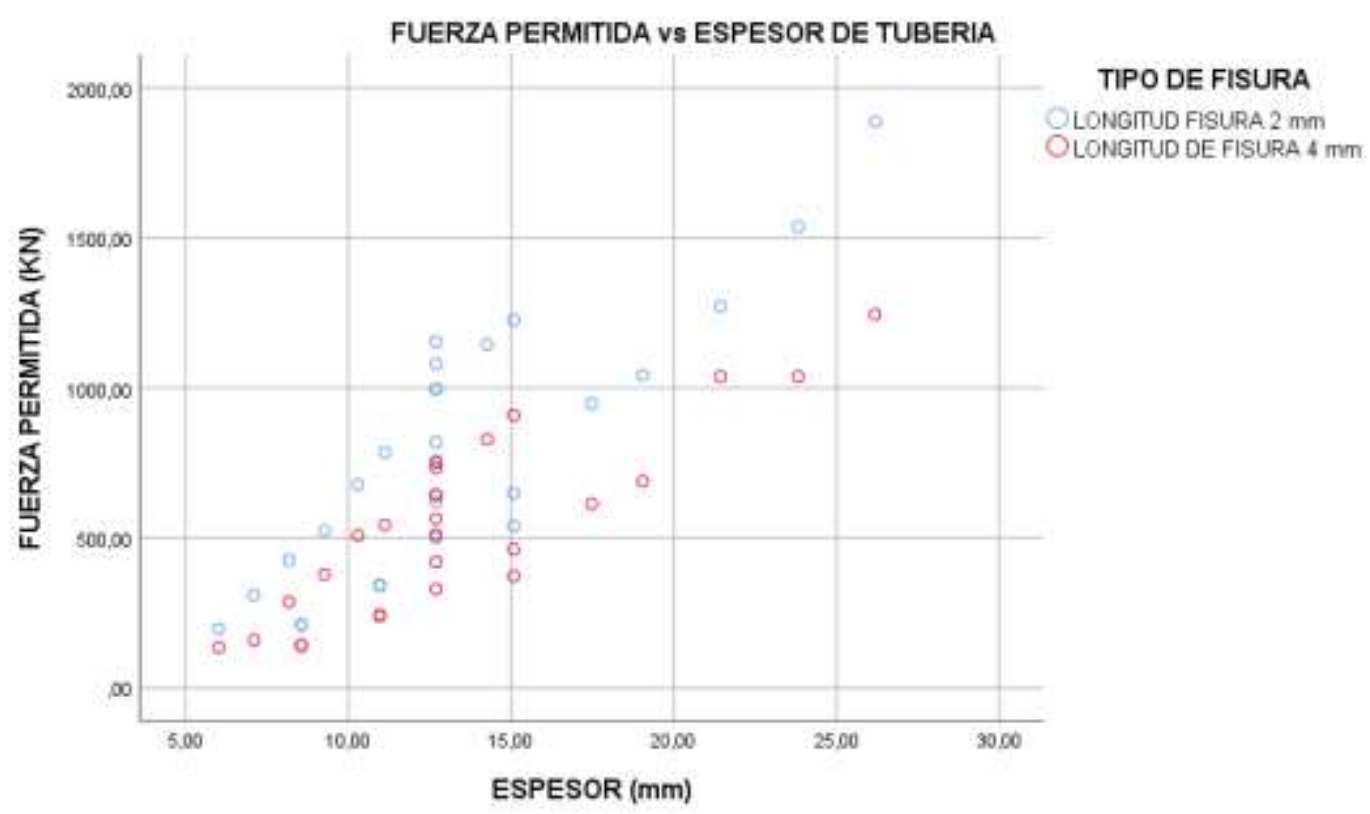

Fuente: Elaboración propia

A medida que el espesor aumenta la Fuerza Permitida crece proporcionalmente, las longitudes de las fisuras juegan un papel importante en predecir la fuerza que se puede aplicar a la junta soldada. Una fisura de mayor longitud es más peligrosa, por ende, la Fuerza permitida es menor.

En la figura 18. se observa le variación que tiene la Fuerza Permitida en cuanto a los valores obtenidos en la simulación con los valores obtenidos mediante el modelo de Regresión Lineal Múltiple. Existe un error significativo en los 7 primeros valores con respecto a las fisuras del tipo 2., incluso obteniendo valores negativos que se alejan de la realidad. Sin embargo, el resto de los valores se apegan al modelo. 


\section{Figura 18}

Fuerza permitida vs número de simulación (fuerza permitida de simulación y del modelo)

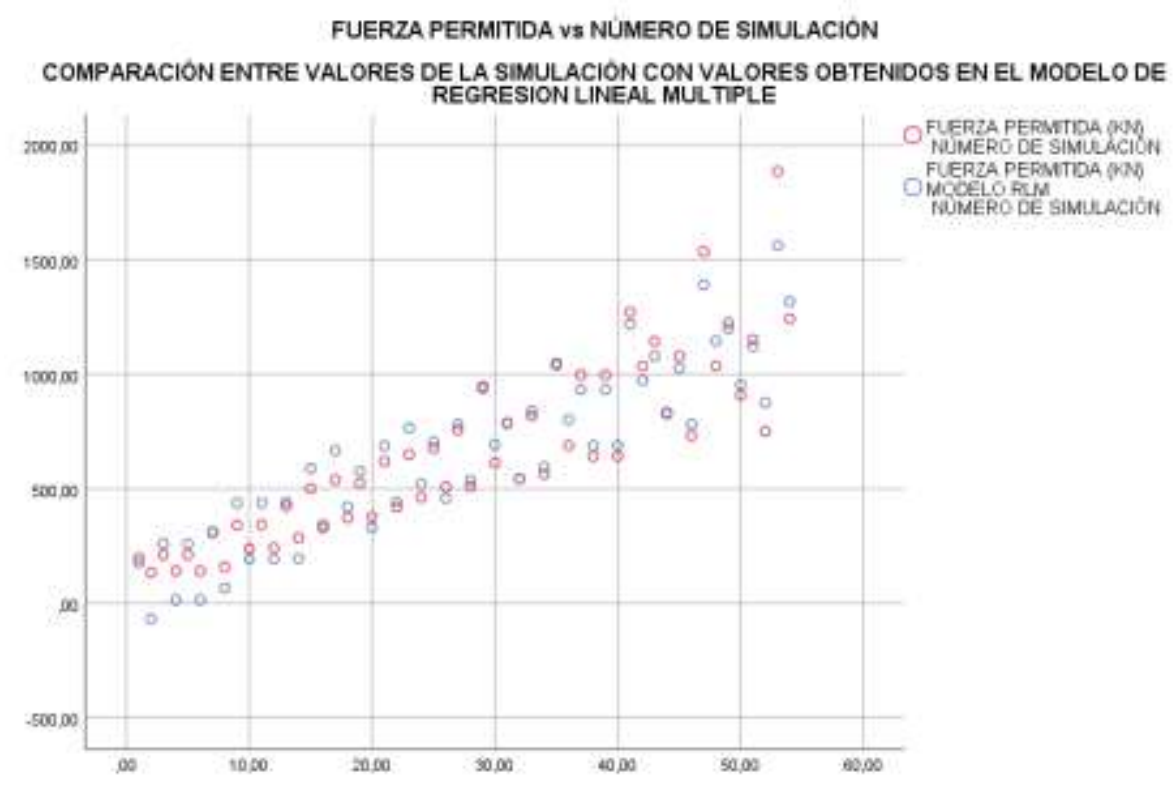

Fuente: Elaboración propia

Finalmente se indica cómo se reduce la fuerza que se puede aplicar a una junta soldada, La dispersión de color rojo muestra la fuera que se ha aplicado a cada tubería dependiendo del diámetro y del espesor, mientras que la dispersión de color azul indica la fuerza permitida que se puede aplicar a la misma tubería, pero con una fisura específica para que el material no falle o supere el límite elástico.

\section{Figura 19}

Reducción de aplicación de la fuerza a tracción (eje vertical - fuerza aplicada y permitida; eje horizontal - diámetro de la tubería)

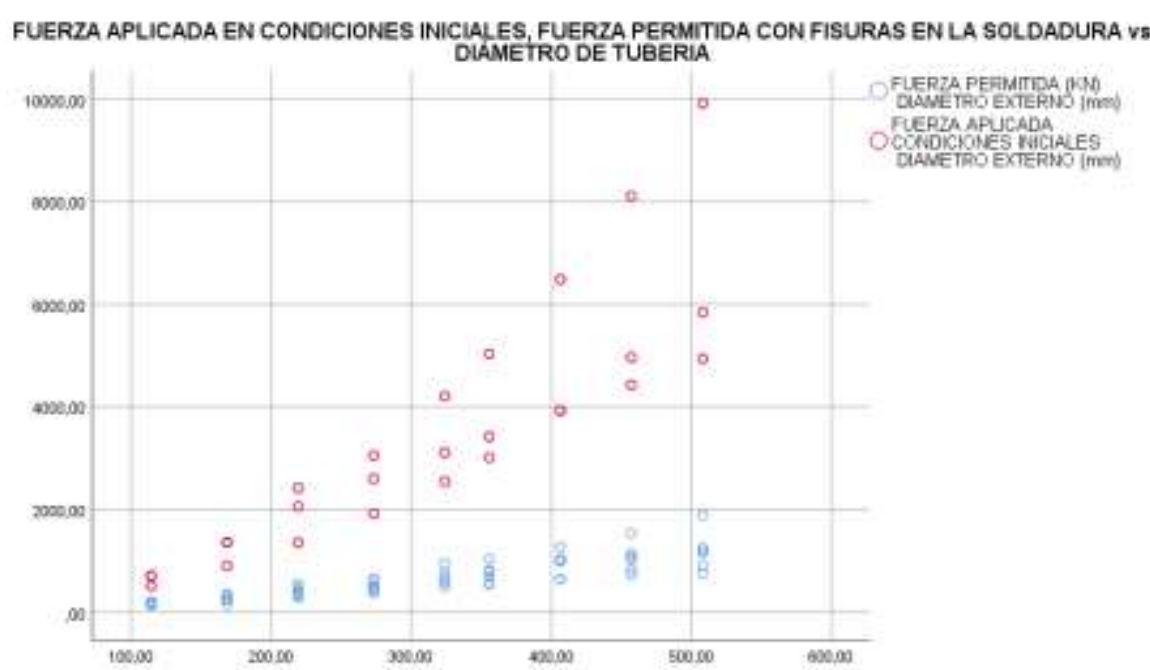

Fuente: Elaboración propia 


\section{Conclusiones}

- El trabajo realizado aborda un problema de campo en la industria petrolera, el de predecir la Fuerza Permitida que se puede aplicar a una junta soldada de tuberías con dos tipos de fisuras, además con la variación de parámetros como el diámetro de una tubería y su espesor. Para determinar la Fuerza Permitida de aplicación se realizó un análisis de concentración de esfuerzos mediante simulación numérica en el software ANSYS obteniendo las siguientes conclusiones:

- Se ha analizado el esfuerzo a tensión de cordones de soldadura con fisuras mediante simulación numérica por elementos finitos, enfocando el análisis en la mayor concentración de esfuerzos que se ha generado en el interior de la fisura en todas las muestras. Este resultado nos ha permitido predecir el porcentaje de reducción de factor de seguridad, indicando el riesgo en forma cuantitativa en la junta cuando se apliquen cargas de tensión.

- Se ha establecido el modelo 3D tanto para las tuberías como para la soldadura, los diámetros y espesores de las tuberías han sido obtenidas de catálogos de empresas distribuidoras de este tipo de elementos, esto con el objetivo de realizar un análisis realista. Se han tomado 9 diámetros nominales desde 4 hasta 20 pulgadas, no se puede realizar de diámetros mayores porque el proceso de manufactura es con una soldadura longitudinal lo cual afectaría al análisis. Los espesores se han ocupado de acuerdo con las cedulas disponibles en el mercado, 40, XS y 80 cada una con diferentes espesores que dependen del diámetro.

- El modelo de las fisuras se ha tomado de forma elíptica y dos longitudes 2 y 4 $\mathrm{mm}$. con una profundidad constante de $1 \mathrm{~mm}$. y espesor de $0,5 \mathrm{~mm}$. también constante. Cabe señalar que el recurso computacional es limitado para la realización de las 54 simulaciones realizadas y por ende no se ha podido introducir longitudes mayores en cuanto a fisuras.

- La resistencia a la tracción se ha evaluado aplicando un esfuerzo en sentido longitudinal a la tubería con un valor constante de $250 \mathrm{MPa}$. Este valor se obtuvo a partir del límite elástico del material utilizado ASTM A36, esto significa que la concentración de esfuerzo máximo en la fisura deberá ser 1 para que no falle el material elásticamente sin que haya deformación permanente. Pero esto no ocurre si se tiene una discontinuidad en la junta (Fisura), evidenciando una concentración de esfuerzos muy elevados y reduciendo el factor de seguridad, poniendo en riesgo el sistema de unión. Para que la simulación sea válida se ha programado un bucle de refinamiento de malla hasta que el resultado final tenga un error menor al $10 \%$ con el penúltimo realizado, garantizando una convergencia y un resultado real. Estos resultados son procesados a fin de tener un valor descifrable en cuanto a que fuerza máxima puedo aplicar a una tubería con fisuras si el factor de seguridad se ha reducido.

- Al tener una variable dependiente (FUERZA PERMITIDA) y 3 variables independientes (Diámetro de tubería, espesor y longitud de fisura) se ha seleccionado una técnica multivariante explicativa como la regresión lineal 
múltiple, a fin de identificar las variables de mayor correlación, mayor influencia sobre la variable dependiente y la ecuación de modelo que describa el fenómeno.

- La variable diámetro de la tubería tiene la mayor correlación de 0.868 con la variable dependiente, indicando una relación proporcional, a mayor diámetro de tubería mayor es la fuerza a tracción que se puede aplicar sin que falle el material hasta el límite elástico. Esto se debe a que se tiene una mayor longitud de cordón de soldadura con respecto a la longitud de la fisura.

- Como segunda variable independiente es el tipo de fisura, la lógica es fundamentada por el análisis de los datos de las simulaciones, mostrando una tendencia de proporcionalidad con la fuerza permitida a tracción que se puede aplicar, las fisuras tipo 1, de longitud $2 \mathrm{~mm}$ presentan menor concentración de tensiones, lo cual puede soportar la junta mayor fuerza de tracción, mientras que las fisuras tipo 2 de $4 \mathrm{~mm}$ de longitud tienden a presentar mayor riesgo en la unión soldada.

\section{Referencias bibliográficas}

ANSYS. (18 de noviembre de 2019). Ansys help 19.1. Obtenido de Ansys help 19.1: https://ansyshelp.ansys.com/account/secured?returnurl=/Views/Secured/corp/v1 91/wb_msh/msh_Element_Quality_Metric.html

ANSYS RELEASE. (2010). ANSYS Meshing User's Guide. U. S. A.: Ansys, Inc.

API 1104. (2013). Welding of Pipelines and Related Facilities. Washington, DC: American Petroleum Institute.

API 579. (2007). Recommended Practice 579 Fitness for Service. Washington D. C.: American Petroleum Institute.

AWS. (2002). Structural Welding Code - Steel. Miami: LeJeune Road.

Beer, F., Johnston, R., Dewolf, J., \& Mazurek, D. (2012). Mechanics of Materials. New York: McGraw - Hill Companies, Inc.

Chen, X. L. (2014). Finite Element Modeling and Simulation with ANSYS Workbench. New York: Taylor \& Francis Group.

Fiorella Representaciones S.A. (8 de enero de 2018). FIORELLAREPRE. Obtenido de FIORELLAREPRE: https://www.fiorellarepre.com.pe/FichaTecnica/804610.pdf

Jayatilaka. (1979). Fracture of Engineering Brittle Materials. London: Applied Publishers LTD.

Li Y, S. J. (2018). Investigation on size tolerance of pore defect. PLOS ONE, 1-17.

Massa, J., \& Giudici, A. (2009). Comportamiento de un gasoducto con fisuras. Revista Internacional de Desastres Naturales, Accidentes e infraestructura Civil, 143162. 
Oller, S. (2001). Fractura Mecánica un enfoque global. Barcelona: Artes Gráficas Torres S. A.

Pozo, L., Campillo, M., \& Lascano, S. (2017). Simulación numérica de la presencia de una grieta cercana a la soldadura de una unión tubular en T. Ingeniería y desarrollo, 320-336.

Suarez, F. C. (s.f.). Simulación del proceso de rotura por tracción de alambres de acero pelítico con un modelo de fisura cohesiva. In Anales de Mecánica de la Fractura, 207-212.

Yépez, H. (2015). Análisis de mecánica de fractura en tuberías soldadas de gaseoducto mediante simulación numérica por elementos finitos. Lima: PUCE.

Zerbst, U., Heerens, J., \& Schwalbe, H. (2002). The fracture behavior of a welded tubular joint - an ESIS TC1.3 round robin on failure assessment methods Part I: experimental data base a summary of results. Engineering Fracture Mechanics, 1111-1118.

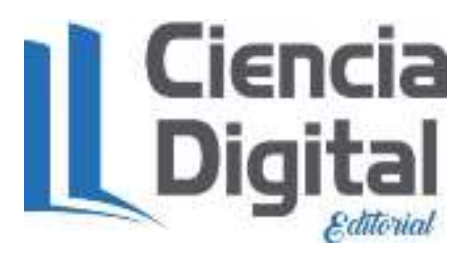




\section{PARA CITAR EL ARTÍCULO INDEXADO.}

Serrano Aguiar, C. O., Calispa Aguilar, M. E., Ordoñez Viñan, M. A., \& Choto Chariguamán, L. S. (2021). Efecto de fisuras en la tracción de juntas soldadas de acero A36 mediante simulación numérica. ConcienciaDigital, 4(4.2), 77-100. https://doi.org/10.33262/concienciadigital.v4i4.2.1948

\section{LCiencia}

El artículo que se publica es de exclusiva responsabilidad de los autores y no necesariamente reflejan el pensamiento de la Revista Conciencia Digital.

El artículo queda en propiedad de la revista y, por tanto, su publicación parcial y/o total en otro medio tiene que ser autorizado por el director de la Revista Conciencia Digital.

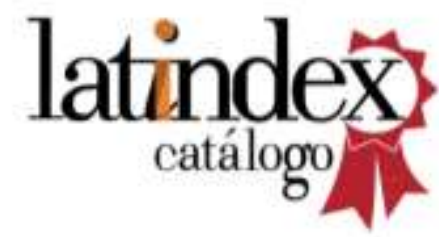

